\title{
Research of Trajectory Generation of Robot Based on CAD File Zeng Min
}

\author{
(NanChang Institute of Science \& Technology, Nanchang,330108; Jiangxi Engineering Laboratory \\ of Digital Manufacturing for Automobile Parts)
}

\section{Keywords: CAD; Industrial Robot; Trajectory Planning; Research}

\begin{abstract}
Trajectory planning of robot has the important position in the control of robot. Taking the industrial robot for research objects, this paper carried out the continuous path planning for the robot end-effector, proposed the continuous path algorithm of robot based on CAD/CAM technology and realized NC machining simulation through the modeling technology of virtual robot. Finally, the analysis and experiments showed that the continuous path movement generated by the algorithm can satisfy the process requirements of complex machine with high speed and high precision because of higher precision and stabler operation. And then, on the basis of VERICUT, through modeling of virtual robot and modifying the parameter file and control file of robot, NC code generated in CAD/CAM system can be directly used to drive the robot for completion of simulation, and the whole process of simulation processing of robot is showed through a practical example.
\end{abstract}

\section{Introduction}

When the industrial robot is used in path tracing applications such as welding, cutting and flame spraying, the movement of end effector must follow the special trajectory in three dimensional space at the speed as rated as possible. In the following cases, when the speed, nodal acceleration and trajectory error of the end effector are limited, the trajectory planning may be very complex. For the trajectory planning without full consideration of these limitations, it often get bad results such as overshoot trajectory, deviation of the end effector from the given trajectory and excessive velocity fluctuation. Aiming at the basic features of robot, this paper proposed a new trajectory planning algorithm that the NC machining simulation is realized with help of modeling technology of virtual robot to make NC code generated in CAD/CAM system to directly drive the robot for completion of simulation, and the whole process of simulation processing of robot is shown through a practical example.

\section{Concept of Trajectory Planning}

Robot trajectory is generally refer to the movement trajectory of industrial robot during exercise, i.e., the displacement, speed and acceleration of the moving point. Trajectory planning is refer to calculation for the anticipated movement track according to the requirement of tasks, i.e., describing the task, movement path and track of robot, calculating the displacement, speed and acceleration of robot's movement in a real time and generating the movement track. The trajectory planning of industrial robot belongs to the bottom planning of robot which basically does not involving artificial intelligence. Trajectory planning can be carried out in both joint space and cartesian space.

When the trajectory planning is carried out in the cartesian space, the end effector of robot is used to plan the trajectory of function for definition in the cartesian space, it make the gripper\&posture, speed and acceleration to be represented by a time function. The corresponding joint position, speed and acceleration are educed by the hand claw information. Because of joint actuation, the space constraint must be converted to the joint, and a certain function shall be used to describe and generate one trajectory to satisfy the joint restriction, and make it to be as close to the spatial path as possible.

Trajectory planning method is generally refer to that a series of "control setting points" are generated by adding the interpolated points and the approaching points relative to the given path between the initial position and the target position of robot. The giving way of target trajectory 
includes teaching playback and numerical control.

Whether the teaching playback or the numerical control, it is required to generate the target trajectory between points and points. This target trajectory is generated according to different conditions and requirements, but some common principles must be followed.

\section{Conversion of Joints of Robot}

Transforming the interpolation points obtained from the Cartesian coordinate into the interpolation points in joints coordinate system, the joint variables of all interpolation points on the robot trajectory can be calculated in accordance with the reversal kinematic formula. To get the exact planned trajectory, we will carry out the trajectory planning in the cartesian space. If the end effector of robot want to move to the desired position, it must be clear to know the postures of link rods correspond to the points on the path. The determination of posture of the end link rod is required for the postures of all link rods. And the corresponding variables correspond to the joints are obtained according to knowledge of reverse kinematics. Therefore, posture of the end link rod is the key of solution. As required, the end effector is planned to be perpendicular to the operating platform, and the posture is obtained.

The operating speed of robot shall be firstly planned for calculation of operation time of robot. The operation speed of the end link rod of robot is planned to be a constant speed $20 \mathrm{~mm} / \mathrm{s}$, and the operation time of whole trajectory is the sum of the operation time of each trajectory. The choice of speed of robot $(\mathrm{mm} / \mathrm{s})$ :

In the robot controller, we can set the default as the speed of robot, and also can customize the speed of robot. The maximum speed can be set as v7000 which may not be achieved in the practical operation. For calculation of the joints variables of all trajectories of robot, the expression for joints variables based on the reversal kinematic of robot and MATLAB software are required.

\section{Trajectory Planning System for Robot}

The trajectory planning of robot includes two aspects which are optimization for the path of robot (i.e., space) and optimization for the moment that the robot move to the described position (i.e., time optimization). the purpose of trajectory planning is to make the machine hand (end effector) to move on the given trajectory more smoothly. The start, turning point and end are the movement points of discontinuity. At this time, the impulsive force may be very strong, even the acceleration may instant become infinite. It is ideal to set the acceleration of the machine hand as zero at these positions. Therefore, we should make the acceleration of robot to be as low as possible at these positions to protect the robot. According to the work space of robot, the path of robot is planned to avoid singular point and the case of exceeding the work space of robot as following.

This paper adopted the spline curves of CAD/CAM system. It may represent the analytical curve (as straight line, conical section, etc.) and the free curve (as uniform B-spline curve, etc.) accurately by uniform algebraic expression. Therefore, it is convenient for uniform database management and storage, and the program code size can be greatly reduced. The parameter form is used to represent curve in most of CAD/CAM systems.

The specific process of extracting coordinates from $\mathrm{G}$ code obtained is as follows:

With N001 G01 X10 Y20 as an example:

(1) Extract the single key character such as "N" and " $G$ ";

(2) Match it to the characters in the character library constructed, next step if matched;

(3) Extract the number after character and store it in a buffer;

(4) Store the number obtained in the specified file.

The specific flow of planning system for robot is shown in Figure 1: 


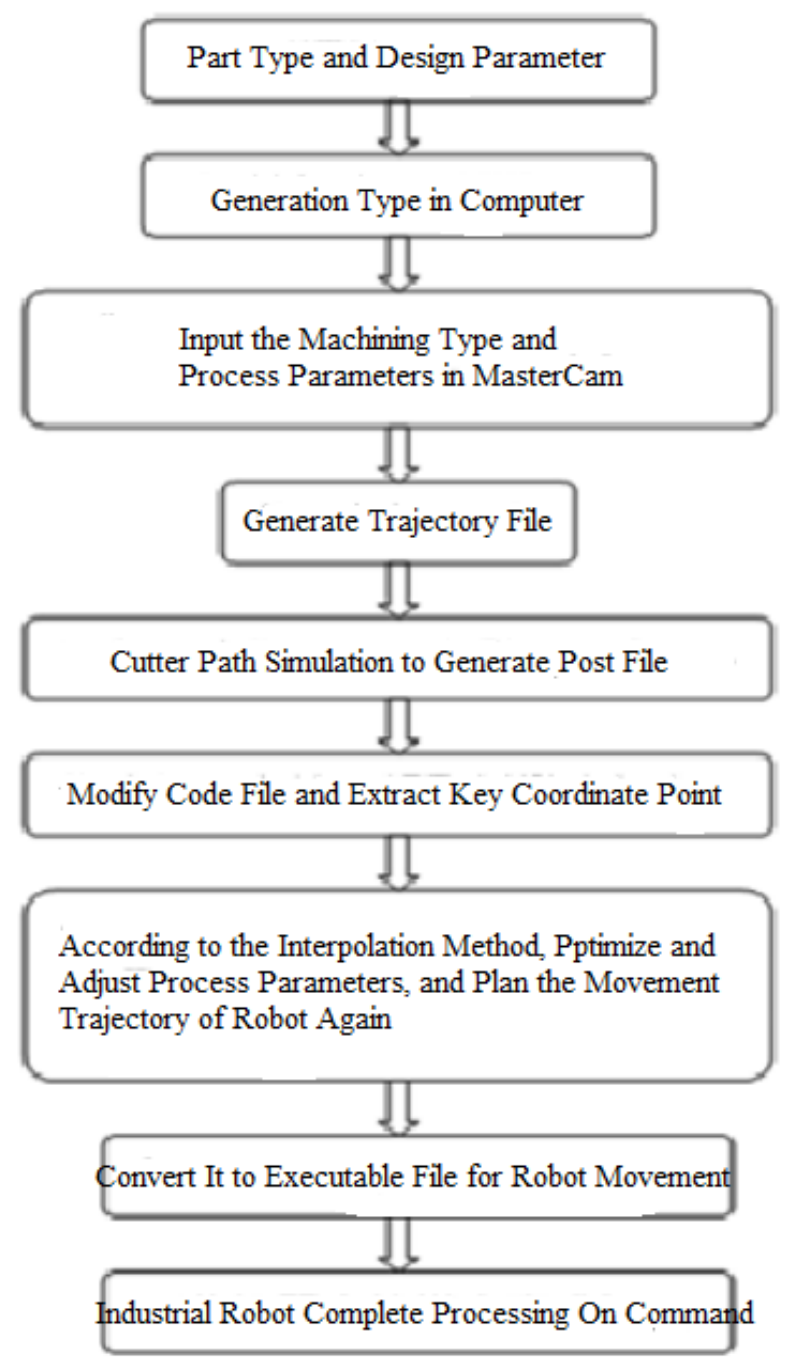

Figure 1. Continuous Path Planning System of Robot Based on CAD/CAM

Through optimization for parameters that the movement trajectory obtained from CAD/CAM is optimized by replacing the parameter $\mathrm{u}$ with accumulative chord length $\mathrm{s}$, the movement trajectory of robot is planed again to realize the information flow between computer aided design\&manufacturing and robot system. The specific interpolation method is as follows: obtain $\mathrm{NC}$ code of processing profile through CAD/CAM; extract the features; fit the features to many sections of cubic spline curves; carry out the uniform interpolation for the sections of cubic spline curves; obtain the final interpolation point. The coordinates of posture interpolation of robot are obtained by carrying out the posture interpolation of robot according to the value of the interpolation point obtained by the robot. Lastly, with the reverse kinematics function for robot, the rotation angle values of all joints movements of robot are obtained according to the obtained position coordinates and the posture coordinates of robot.

\section{Conclusion}

As a very important and complex problem in robotics, the trajectory planning of robot has long been the keystone and difficulty of the study of robot. This paper mainly studied the continuous path of industrial robot. Based on the research work for robot, this paper proposed the continuous path planning algorithm for robot based on CAD/CAM technology that the further research is carried out based on the existing CAD/CAM system to make NC code generated in CAD/CAM system to directly drive the robot for completion of simulation, and the whole process of simulation processing of robot is shown through a practical example. This algorithm can help to resolve all 
problems existed in the actual processing on a computer, reduce the development cycle of product, lower the production cost and improve the quality of products.

\section{Acknowledgement}

Project funding: Project at Nanchang institute of science and technology (No.SZZX-17-25)

\section{References}

[1] Chen H, Sheng W, Xi N, et al. CAD - based automated robot trajectory planning for spray painting of free - form surfaces[J]. Industrial Robot, 2002, 29(5):426-433.

[2] Sobh T M, Wang B, Patel S. A Mobile Wireless and Web-based Analysis Tool for Robot Design and Dynamic Control Simulation from Task Points Description[J]. Journal of Internet Technology, 2003, 4(3):153-161.

[3] Song H C, Song J B. Precision robotic deburring based on force control for arbitrarily shaped workpiece using CAD model matching[J]. International Journal of Precision Engineering \& Manufacturing, 2013, 14(1):85-91.

[4] Dani J, Winkler A, Suchy J. Robot Programming for Surface Finishing based on CAD Model Including External Axes[C]// Robotics; Proceedings of Robotik 2012; German Conference on. VDE, 2012:1-6.

[5] Xu J N, Hu Z X, Weng L X. Mechanical Cutting Process of Cutting Robot Based on CAD/CAM Integrated System[J]. Applied Mechanics \& Materials, 2014, 716-717:1666-1670.

[6] Liu C Y, Yang X B, Wang Z Z, et al. Trajectory generation of painting gun based on Z-map theory[J]. Machinery Design \& Manufacture, 2012.

[7] Lin F Y. Path Generation for Robot Polishing System Based on Cutter Location Data[J]. Advanced Materials Research, 2014, 902:250-253.

[8] Zhou B, Zhang X, Meng Z, et al. Off-line programming system of industrial robot for spraying manufacturing optimization[C]// Control Conference. IEEE, 2014:8495-8500.

[9] Song H C, Kim B S, Song J B. Tool path generation based on matching between teaching points and $\mathrm{CAD}$ model for robotic deburring[C]// Ieee/asme International Conference on Advanced Intelligent Mechatronics. IEEE, 2012:890-895.

[10] Khelifa B, Ryad C, Toufik B, et al. Virtual Reality Based Tool for Optimal Robot Placement in Robotized Site Based on CAD's Application Programming Interface[C]// Viric. 2010.

[11] Reddy A C, Reddy P R, Kotiveerachari B. Studies on the effect of vibration of robot links on the end point trajectory path using $\mathrm{CAD}[\mathrm{C}] / /$ International Conference on Recent Advances in Material Processing Technology. 2015.

[12] Chen S, Fan $\mathrm{X}$, Li $\mathrm{H}$, et al. Trajectory planning for five-axis welding robot based on CAD/CAM technology[J]. Modern Manufacturing Engineering, 2017. 